\title{
Design of a Novel Non-Invasive Wearable Device for Array Surface Electromyogram
}

\author{
Wenguang Jin, Yidong Li, and Shangyao Lin
}

\begin{abstract}
This paper describes the design of a novel non-invasive wearable device that acquires 128-channels array surface electromyogram (sEMG) in real-time. The device contains 8 individual microcontroller-based acquisition modules and a data fusion module. The acquisition modules share the same structure, and the data fusion module is an ARM-based platform running the Linux operating system. A 16-channel matrix-type $(2 \times 8)$ electrode array patch is used in each acquisition module to parallel acquire sEMG signals, which are digitalised by 2 ADCs. Every $100 \mathrm{~ms}$, the sEMG from 8 acquisition modules are packed in the ARM controller and transferred to PC via WIFI. The SEMG signals are filtered with infinite impulse response recursive least-squares adaptive filter (IIR RLS) on PC.
\end{abstract}

Index Terms-Surface electrode array, sEMG, signal acquisition, wearable device.

\section{INTRODUCTION}

The sEMG is the tiny electrical signal generated by nerves when muscle acts and detected on the skin by non-invasive measurement technique. It reflects the activity of muscle [1]. The sEMG is widely used for clinical rehabilitation [2] athlete training [3] and human-computer interaction (e.g. gesture recognition [4]). Compared with indwelling or intramuscular electromyogram signals (iEMG), sEMG is non-invasive and much easier to operate.

The sEMG can be detected via surface electrode. There is rich information in sEMG, including space and time information of the muscular contraction and relaxation [5]. Zhao et al. implemented an acquisition system of sEMG [6]. But his system detected very few channels. It had a limit to the number of gestures to be recognised. Therefore, it is a trend to focus on the research of multi-channel sEMG. Lapatki et al. used a thin, flexible multi-electrode grid for high-density sEMG [7]. Nagata et al. designed a 96-channels sEMG acquisition system based on surface multielectrode [8] and developed an sEMG recognition algorithm [9]. These researches were based on traditional signal processing methods.

In this paper, a new wearable device is implemented. It acquires 128-channels array sEMG as a matrix of $16 \times 8$ in real-time. It can be viewed as a two-dimension grey-scale image. By image processing approaches, the relations among

Manuscript received October 4, 2014; revised October 11, 2015. The research work was supported by National High-tech Research and Development Program ("863" Program) of China under Grant No. 2013AA013705.

The authors are with the College of Information Science and Electronic Engineering, Zhejiang University, China (e-mail: jinguang@zju.edu.cn, pzdongdong@163.com, zjulsy@zju.edu.cn). different muscle sEMG may be processed generally. The cross-talk may also be ignored.

\section{MATERIALS AND METHODS}

In our research, a device acquiring array sEMG is implemented. It is designed to be wearable, flexible and non-invasive. Fig. 1 presents the general structure of the device which includes 8 same acquisition modules and 1 data fusion module. Fig. 2 shows the structure of each acquisition module. Each acquisition module can get 16-channel matrix-type $(2 \times 8)$ sEMG signals. Then, 128-channels array sEMG from 8 acquisition modules are transferred to the data fusion module, which is an ARM controller platform. Finally, the matrix-type $(16 \times 8)$ data are transmitted to PC via WIFI to be processed.

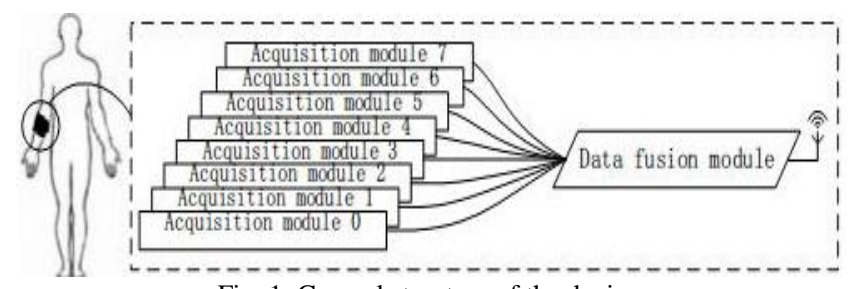

Fig. 1. General structure of the device.

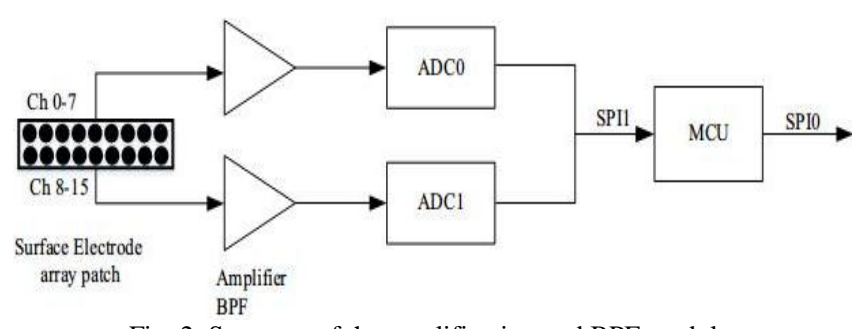

Fig. 2. Structure of the amplification and BPF module.

\section{A. Suface Electrode Array Patch}

The surface electrode array patch is one of the features of our device. It is also the key to get array sEMG. Fig. 3 is the surface electrode array patch, which contains 18 electrodes ( 2 $\times 9$ ). Each electrode has a diameter of $3 \mathrm{~mm}$ and is arranged with an interval of $8 \mathrm{~mm}$ in horizontal and $8.5 \mathrm{~mm}$ in vertical.

We use polyethylene terephthalate (PET) as the base of the patch to make it flexible and harmless to skin. Except for the electrodes and the interfaces at the left and right side, the patch is insulated. Up on the PET base is medical double-side tape with which the patch can be fixed on the skin. The material of electrodes and wires is Ag. Conductive gel covers the silver electrodes. The contact impedance of the electrodes is measured less than $3 \mathrm{~K} \Omega$. The materials and the short path improve the contact with the skin and the signal quality. 
As shown in Fig. 3, one channel's signal is a differential signal converted from the raw sEMG signals of two neighbour electrodes in the same line. There are 16 channels in 1 patch. Each of the 8 acquisition modules owns a surface electrode array patch. Thus, the whole device can detect 128 channels.

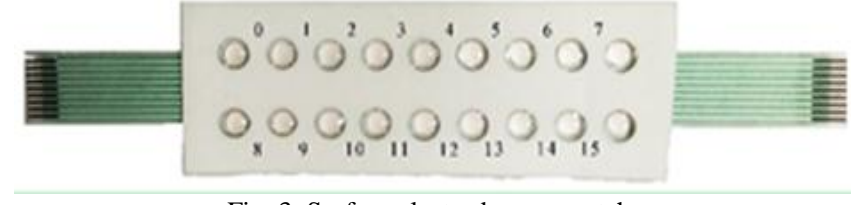

Fig. 3. Surface electrode array patch.

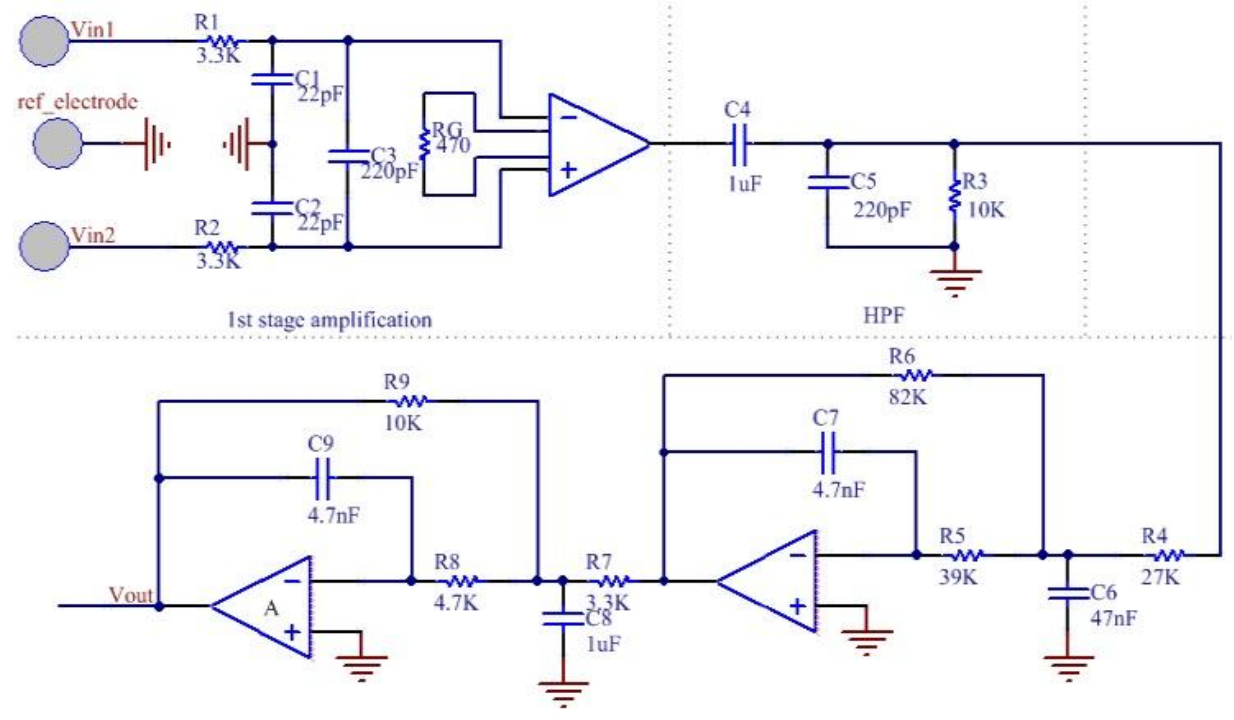

2nd stage amplifiation and LPF

Fig. 4. Circuit diagram of amplification and BPF module for 1 channel.

\section{B. Amplification and BPF}

The differential signal is amplified and filtered in the amplification and band-pass filter (BPF) module (Fig. 4).

As the raw sEMG amplitude is very tiny, varying from 0 to $10 \mathrm{mV}$, an amplification circuit with a gain of $60 \mathrm{~dB}$ should be applied to make it possible for analog-to-digital conversion. Considering the large gain, this amplification circuit contains two stages. Besides, a BPF is necessary because the frequency band is mainly limited from $20 \mathrm{~Hz}$ to $500 \mathrm{~Hz}[10]$.

In the first stage, the monolithic instrument amplifier of Analog Devices' AD8224 is the main element. The low-cost and micro-power AD8224 has high CMRR (100 dB), low electronic noise $(14 \mathrm{nV} / \sqrt{\mathrm{Hz}})$, low input bias current $(<10$ $\mathrm{pA})$, high input impedance $\left(10^{4} \mathrm{G} \Omega\right)$ and rail to rail output. Amplifier with high CMRR has better ability to suppress power line interference. Ag electrodes stay stable longer with low input bias current. More gain is allocated to the first stage to reduce noise. The resistor $\mathrm{RG}$ is selected to obtain the amplification coefficient of $40 \mathrm{~dB}$. The value of $\mathrm{RG}$ is determined by equation below:

$$
\mathrm{RG}=\frac{1+49.4 \mathrm{~K} \Omega}{\text { Gain }} \approx 490 \Omega
$$

The second stage is a BPF. At first, a high-pass filter (HPF) with $20 \mathrm{~Hz}$ of cut-off frequency is used to reject artifact and remove the dc drift. Then a 4th order Chebyshev LPF is applied to avoid high-frequency noise, its gain is $20 \mathrm{~dB}$ and cut-off frequency is $380 \mathrm{~Hz}$. The 2 low cost micropower, low noise CMOS operational amplifiers used here are Analog Devices' AD8617. It has high CMRR (> $68 \mathrm{~dB}$ ), low electronic noise $(22 \mathrm{nV} / \sqrt{\mathrm{Hz}})$, low input bias current $(<1 \mathrm{pA})$ and rail-to-rail input and output.

After the $2^{\text {nd }}$ stage, the signal amplitude is amplified by 1000 times, the frequency is limited between $20 \mathrm{~Hz}$ and 380 $\mathrm{Hz}$, and the dc drift is removed.

\section{Analog-to-Digital Conversion and Microcontroller}

After the amplification and BPF filter, the sEMG signal is digitalised by the low-power ADC of Texas Instrument's ADS1198. It provides high resolution data of 16 bits, programmable gain, and 8-channel parallel data. The parallel data guarantee the sEMG of different channels is synchronised. The ADC uses a continuous conversion loop and its sampling rate is $1 \mathrm{KHz}$. This sampling rate is sufficient for acquiring sEMG signals by Nyquist sampling theory.

In one acquisition module, there are two ADCs. Each ADC acquires 8-channel signals. As shown in Fig. 2, signals of Channel 0-7 are digitalised by ADC0, signals of channel 8-15 are digitalised by ADC1. The two ADCs are controlled by a low-power and low-cost MCU (Freescale MKL25Z). The MCU's core frequency is $48 \mathrm{MHz}$. The two ADCs share one ready signal because they work synchronously. Every $1 \mathrm{~ms}$, ADC0 sends a ready signal to $\mathrm{MCU}$, and the MCU reads the two ADCs' data in order via SPI (4 Mbps). Then it processes the data and waits for the data fusion module to read them via another SPI (4 Mbps).

\section{Data Fusion Module}

The data fusion module contains an ARM controller of Samsung S3C6410 and a WIFI controller of Marvell 88W886. The ARM controller's clock frequency is $667 \mathrm{MHz}$. An embedded Linux operating system is running on it. The software we developed controls the ARM controller to offer 8 
acquisition modules synchronization signal and read 8 modules data every $100 \mathrm{~ms}$ via multiplexed SPI (4 Mbps). These data are composed as 100 matrices $(16 \times 8)$. The WIFI module, supporting TCP/IP protocol and having a maximum rate of $54 \mathrm{Mbps}$, sends all the data to PC per $100 \mathrm{~ms}$. On PC, our software collects and filters the data, removes the dc drift and plots the real-time signal in time domain. We use an IIR RLS [11] to filter the power line interference.

\section{RESULTS}

Fig. 5 presents our device. (a) is the board of amplification and BPF circuit. The surface electrode array patch is inserted into the bottom of this board. (b) is the board of the ADC and MCU circuit. (c) is the general look of the device. A lithium battery is used as its power supply to reduce power line interference. But the interference from body and air still exists. (d) is the typical way of how the de-vice is worn. First of all, the skin should be cleaned with alcohol. Then, The 8 acquisition modules are worn around the right forearm anticlockwise (the tester faces up to me). A reference electrode is stick to right wrist. The data fusion module and the battery are in the pocket.

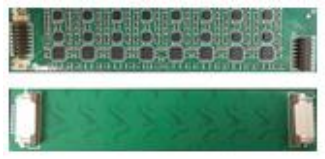

(a)

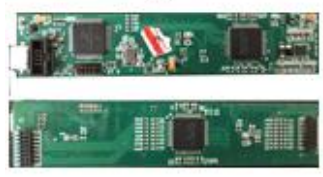

(b)

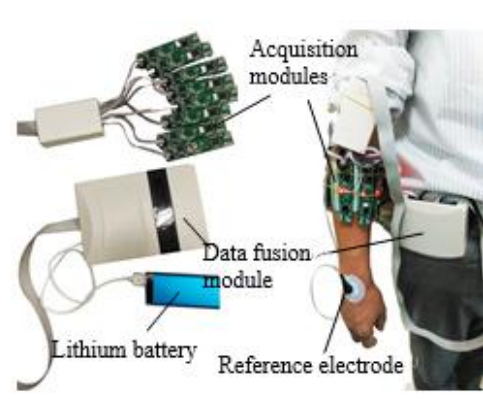

(c) (d)
Fig. 5. (a) Amplification and BPF board (b) ADC and MCU board (c) The general look of the device (d) Device is worn on the right forearm.

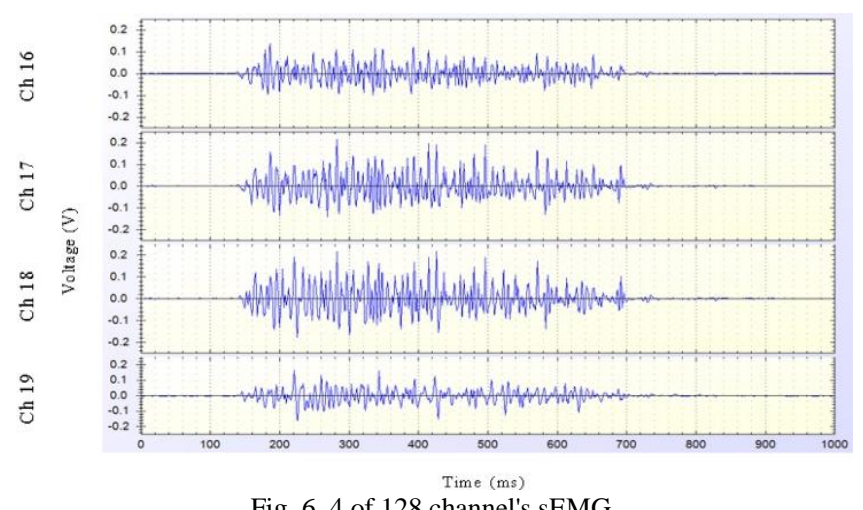

Fig. 6. 4 of 128 channel's sEMG.

We tested the device with healthy volunteers. The 128-channels sEMG signals were successfully acquired simultaneously. Fig. 6 shows the signal time domain plots of the measurement. Our software on PC can display 128-channels signals, 4 of them are presented here. Channels 16-19 are the 4 channels in acquisition module 1 (Fig. 1), which locates at the 11 o'clock position of the forearm. The tester was asked to relax at the beginning. Hearing command, he quickly opened his palm and stretched his fingers. It lasted for about 0.5 second. Then he relaxed again. We told the tester to do it lightly. We can see that the signal amplitude was very clean when the tester was relaxing, and it oscillated between $-0.2 \mathrm{~V}$ and $0.2 \mathrm{~V}$ when the tester acted. The signal could be easily distinguished. Hence, the acquisition device is very sensitive to the change of the signal, and the device has the good ability to suppress the noise.

Fig. 7 lists 5 gestures' sEMG signals of channel 16-19. The illustrations at the top of the figure demonstrate the corresponding gestures. They were acquired from one person in one test. It was obvious that different gestures' sEMG signals were quite different. Firstly, the patterns of different gestures' sEMG signals were different as we saw. Secondly, the peak-to-peak amplitude variety of the $1^{\text {st }}$ gesture was the most significant. And the $2^{\text {nd }}$ gesture's was the least. Actually, the little finger, ring finger and middle finger acted harder, the 4 channels' signals were stronger. Therefore, we could deduce that the thumb's movement had less relationship with this muscle. Thirdly, each gesture's sEMG signals of channel $16^{-18}$ were stronger than that of channel 19 . The electrodes related to channels $16^{-18}$ were close to muscle belly while the electrodes related to channel 19 were close to tendon. In other words, the signal at the muscle belly is stronger than that at tendon.

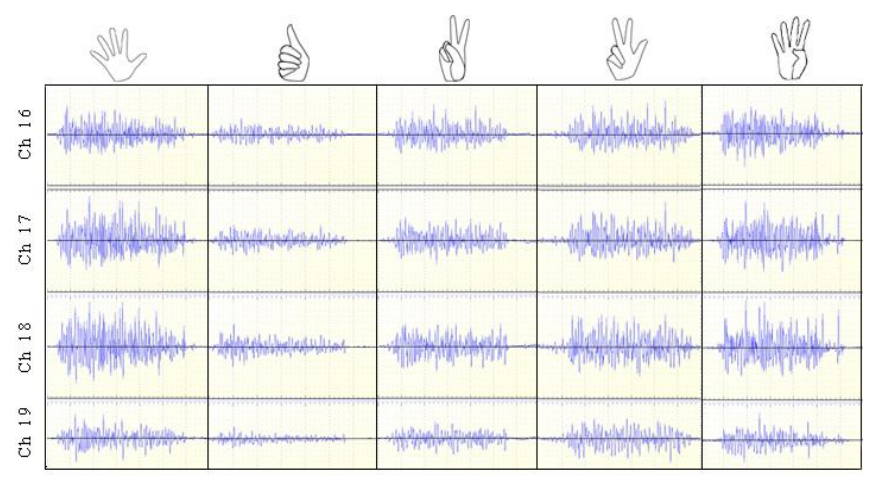

Fig. 7. 4-channel sEMG of 5 gestures.

\section{CONCLUSION}

This paper presents the design of a novel non-invasive wearable device for 128-channels array surface electromyogram. It meets our demand for the real-time measurement of array sEMG. The surface electrode array patch is very important to the quality of the sEMG. Our hardware design and software algorithm effectively remove the dc drift and power line noise. And the device is sensitive to sEMG. Generally, the result is quite satisfying. However, Fig. 6 indicates that the amplitude of the signal is not large enough. If the signal to noise ratio (SNR) can be increased, the signal will be less interfered with the noise.

Our future work will be transplanting the filter algorithm into the device and analysing the signal features with signal and image processing approaches to make it possible to recognise the gestures, so that healthy man without knowing sign language can communicate with the deaf-mute. Besides, we will make the device smaller to be more convenient to wear.

\section{REFERENCES}

[1] R. Song, K. Tong, X. Hu et al., "Assistive control system using continuous myoelectric signal in robot-aided arm training for patients 
after stroke," IEEE Transaction on Neural System and Rehabilitation Engineering, vol. 16, no. 4, pp. 371-379, 2008.

[2] W. Zhu, G. Zheng, Y. Gu et al., "Clinical efficacy and sEMG analysis of a new traditional Chinese medicine therapy in the treatment of spasticity following apoplectic hemiparalysis," Acta Neurologica Belgica, vol. 114, no. 2, pp. 125-129, 2014.

[3] C. Hernandez, E. Estrada, L. Garcia et al., "Traditional sEMG fatigue indicators applied to a real-world sport functional activity: Roundhouse kick," in Proc. the 20th Int. Conf. on Electronics, Communications and Computer, 2010, pp. 154-158.

[4] R. Ganesh, K. Dinesh, P. Sridhar et al., "Independent component approach to the analysis of hand gesture sEMG and facial sEMG," Biomedical Engineering: Applications, Basis and Communications, vol. 20, no. 2, pp. 83-93, 2008.

[5] F. Carlo and C. Paolo, "Multichannel SEMG in clinical gait analysis: A review and state-of-the-art," Clinical Biomechanics, vol. 24, no. 3, pp. 236-245, 2009.

[6] Z. Zhao, X. Chen, X. Zhang et al., "Study on online gesture sEMG recognition," in Proc. the 3rd Int. Conf. on Intelligent Computing, 2007, pp. $1257-1265$.

[7] B. G. Lapatki, J. P. Van Dijk, I. E. Jonas et al., "A thin, flexible multielectrode grid for high-density surface EMG," Journal of Applied Physiology, vol. 96, no. 1, pp. 327-336, 2004.

[8] K. Nagata, K. Ando, S. Nakano et al., "Development of the human interface equipment based on surface EMG employing channel selection method," in Proc. the 28th Ann. Int. IEEE Conf. Engineering in Medicine and Biology, 2006, pp. 6193-6196.

[9] K. Nagata, T. Nakano, K. Magatani et al., "Estimation of muscle strength during motion recognition using multichannel surface EMG signals," in Proc. the 30th Ann. Int. IEEE Conf. Engineering in Medicine and Biology, 2008, pp. 351-354.

[10] J. Chu, I. Moon, and M. Mun, "A real-time EMG pattern recognition system based on linear-nonlinear feature projection for a multifunction myoelectric hand," IEEE Transactions on Biomedical Engineering, vol. 53, no. 11, pp. 2232-2239, 2006.

[11] S. Haykin, Adaptive Filter Theory, 4th ed. Prentice-Hall: Upper Saddle, 2002, pp. 436-467.

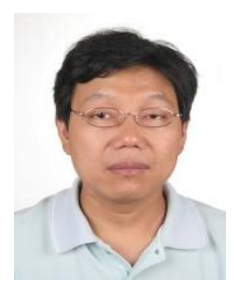

Wenguang Jin was born in 1966, China. He is an associate professor in Zhejiang University, Hangzhou, China. His research interests include wireless communication, mobile embedded system, wireless sensor network, signal acquisition and processing system.

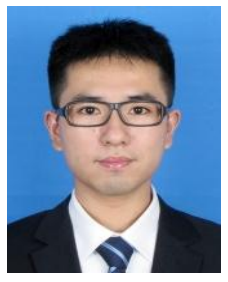

Yidong Li was born in 1991, China. He is now a master candidate in Zhejiang University, Hangzhou, China. His research interests include signal capture, embedded system.

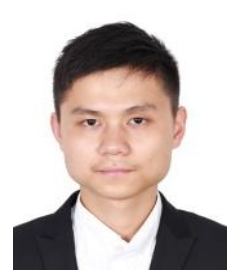

Shangyao Lin was born in 1991, China. He is now a master candidate in Zhejiang University, Hangzhou, China. His research interests include communication protocols, sensor network, and embedded system. 